\title{
The Cochrane Collaboration and the Oral Health Review Group
}

\section{Emma Tavender}

In the first edition of Evidence Based Dentistry P Alderson of the Cochrane Centre in Oxford, UK provided an introduction to the work of the Cochrane Collaboration. He described the contribution made by Archie Cochrane, (1909-1988), a British epidemiologist, and the principles that were established which guided the work of the Collaboration.

Cochrane emphasised that reviews of research evidence must be prepared systematically and they must be kept up-todate to take account of new evidence. If this is not done, important effects of health care (good and bad) will not be identified promptly, and people using the health services will be ill-served as a result. In addition, without systematic up-to-date reviews of previous research, plans for new research will not be well informed. As a result, researchers and funding bodies will miss promising leads, and embark on studies asking questions that have already been answered ${ }^{1}$.

In response to Cochrane's call for systematic, up-to-date reviews of all relevant RCTs of health care, the Cochrane Collaboration was set up in 1992 with the opening of the UK Cochrane Centre under the direction of Dr Iain Chalmers. Just seven years later this centre is just one entity of the rapidly evolving Cochrane Collaboration which now has fifteen Centres world-wide supporting 50 specialist review groups.

This international organisation aims to help people make well informed decisions about health care by preparing, maintaining and ensuring the accessibility of systematic reviews of the effects of healthcare interventions. By definition systematic reviews follow a strict protocol, with clearly defined aims and outcome measures using a specific search mechanism and quality assessment which dictates the eligibility of studies for inclusion in the review. The methodological quality of each study is assessed and the results are presented in a structured report with clearly defined conclusions.

The Cochrane Oral Health Review Group, is one of 50 speciality review groups within the Collaboration. The scope of the group is broadly conceived to include the prevention, treatment and rehabilitation of oral, dental and craniofacial diseases and anomalies. The development of the group is funded by a substantial funding package from the NHS Executive North West, and is based in the Manchester Dental Education Centre of the University Dental Hospital of Manchester, under the coordinating editorship of Professor W C (Bill) Shaw and Dr Helen Worthington.

The group has made considerable progress over the last eighteen months and has two reviews published in The Cochrane Library. Orthodontic treatments for posterior crossbites by Harrison J, Ashby D and Interventions for treating oral lichen planus OLP by Chan E, Thornhill M, Zakrzewska J.

A growing number of registered members has resulted in a steady increase in the number of topics and titles submitted for review. A list of reviews currently being undertaken is published opposite.

In January of this year a highly sensitive search strategy for the group was identified under the guidance of Carol Lefebvre, information specialist at the Cochrane Centre. A specialist register of controlled trials was set up from the results of these searches. Sylvia Bickley, trial search co-ordinator, has continually modified this search strategy to improve the quality of the database which currently holds over 6000 references (who says there is no evidence in dentistry?). This register is undoubtedly the best starting point for anyone considering undertaking a systematic review within the oral health field.

Evidence has shown that searching electronic databases to identify all controlled clinical trials only retrieves about $50 \%$ of the relevant available studies. These reports may either be on the database but inadequately indexed or may never have been entered. To ensure that all RCTs are identified, the Cochrane Collaboration sees handsearching of journals as an important task. The Oral Health Group's handsearching programme is now well established and a small team of handsearchers has been recruited to search the relevant dental journals. Studies identified through handsearching are continually added to the group's specialised register.

The group welcomes all those interested in supporting the work of the group and there are several options for participation, either as principal reviewers, assisting other reviewers, hand searching journals to identify or obtain information on RCTs, or by becoming a member of the panel of peer reviewers. For further details or an information pack contact Emma Tavender, Co-ordinator of the Oral Health Group at: The Cochrane Suite, MANDEC, University Dental Hospital of Manchester, Higher Cambridge Street, Manchester M15 6FH Tel: 01612757818 Fax: 01612757815

\section{The Cochrane Library}

The Cochrane Library is the best source of reliable evidence about the effects of health care for people providing and receiving care, and for those responsible for research, teaching, funding and 
administration at all levels. The Library, published quarterly, is available on compact disc and includes; the Cochrane Database of Systematic Reviews; The Database of Abstracts of Reviews of Effectiveness and The Cochrane Controlled Trials Register. Also included is The Cochrane Review Methodology Database, a handbook on critical appraisal and the science of reviewing research, a glossary of methodological terms, contact details for specialist collaborative review groups and other entities in the Cochrane Collaboration, and Netting the Evidence information on the Internet on using evidence in practice. The Cochrane Library is available in most university libraries and postgraduate medical libraries and can be obtained through personal subscription. Details of subscribing to the Cochrane Library can be obtained from Update Software, PO Box 696, Oxford, OX2 7YX Tel: 01865513902 Fax: 01865516918.

1 Antman E M, Lau J, Kupelnick B, Mosteller F, Chalmers TC. A Comparison of results of meta-analyses of randomized control trials and recommendations of clinical experts. JAMA 1992; 268: 240-8

\section{Cochrane Oral Health Group}

\section{Completed reviews}

Orthodontic treatments for posterior crossbites

by Harrison J, Ashby D

Interventions for treating oral

lichen planus

by Chan E, Thornhill M,

ZakrzewskaJ

\section{Registered Protocols}

Oral care for patients with cancer treated with chemotherapy (excluding head \& neck cancer) by Clarkson J, Worthington $H \mathrm{~V}$, Eden O, Sloan P

Topical fluoride for preventing dental caries in children and adolescents

by Marinho V, Sheiham A, Logan S, Higgins JP T

Potassium nitrate-containing toothpaste for dentin hypersensitivity by Hovgaard O, Poulsen $S$, Errboe M

\section{Registered Protocols (out to referees)}

Guided tissue regeneration for periodontal infra-bony defects by Needleman I, Giedrys-Leeper E, Tucker $R$

Comparison of F-varnishes and sealants in caries prevention by Ahoruo-Saloranta A, Hiiri A, Nordbald A, Mákelá $H$,

Murtomaa $H$

Sealants for caries prevention by Ahoruo-Saloranta A, Hiiri A, Nordbald A, Mákelá $H$,

Murtomaa $H$

Osseointegrated oral implants for tooth replacements by Esposito M, Thomson P Interventions for treating oral leukoplakia by Carrassi A, Sardella A, Lodi G

\section{Registered Titles}

Effectiveness of electric toothbrushes by Shaw W C

Efficacy of anti-calculus dentifrices by Gopalakrishnan NS, Sheiham A
Pain control following third molar surgery in the day-care and out patient setting by Joshi A, Rood P

Scale and polish for chronic periodontitis

by Elley K, Buries A, Gould L Therapeutic trials for recurrent (aphthous) oral ulcers by Chan E, Thornhill $M$, Zakrzewska J

\section{Review currently under discussion}

The effectiveness of early orthodontic treatment by Harrison J E, O'Brien $K$, Worthington $H V$, Lennon $M A$, Williamson $P$

Retinoid therapy for oral cancer lesions

by Thornhill $M$

Facial growth outcome following surgical repair of cleft lip and palate

by Shaw W C

Attitudes, knowledge and behaviour of dental practitioners to infection control guidelines

by Burke T, Bagg J, Grimshaw J 